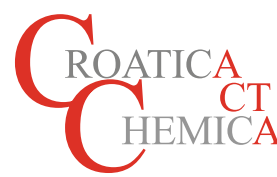

\title{
Computing Degeneracies of the Most Important Structures of Polycyclic Aromatic Hydrocarbons
}

(1) Tomislav Došlić

\author{
Faculty of Civil Engineering, University of Zagreb, Zagreb, Croatia \\ Faculty of Information Studies, Novo Mesto, Slovenia \\ Author's e-mail address: doslic@grad.hr
}

RECEIVED: April 4, 2021 * REVISED: May 5, 2021 * ACCEPTED: May 11, 2021

— This PaPer is Dedicated to Prof. Milan Randić on the ocCasion of his 90 ${ }^{\text {Th }}$ BiRThday, AND to the Memory of Prof. Mircea Diudea —

Abstract: We construct bijections between certain energetically favorable resonance-like structures in several classes of benzenoid compounds and some well-known combinatorial structures enumerated by Catalan and related numbers. In that way we derive explicit formulas for the number of such structures in the considered classes of compounds. We also list some open problems and indicate some possible directions for future research.

Keywords: benzenoid graphs, perfect matching, Kekulé structure, degeneracy, Dyck path, Narayana numbers.

\section{INTRODUCTION}

C HEMISTRY and enumerative combinatorics have been for a long time engaged in a deep and fruitful interaction that plays a significant role in the advancement of both fields. It goes well beyond the standard practice of applying tools from one discipline to the problems of interest in the other. Instead, whole areas of either field benefited from contributions of researchers working on problems of interest to the other one. Good examples are, e.g., the Pólya theory and the theory of matchings in graphs which owe their development in a large part to chemically motivated problems. Matchings, in particular, provide a natural mathematical context for modeling electronic interactions in organic compounds, with perfect matchings serving as models of resonance structures in such compounds.

Our goal in this paper is to derive explicit expressions for the number of certain resonance-like structures in several classes of polycyclic aromatic hydrocarbons. The motivation comes from a recent paper concerned by such structures, ${ }^{[17]}$ but the results could be also relevant in a wider context of matching theory, in particular with respect to the matchings with a given defect number.
In the next section we introduce the relevant concepts and cite some preliminary results. Section 3 contains our main results - explicit formulas for the number of considered structures in several classes of benzenoid graphs. In the last section we summarize our results and indicate some open problems and some possible directions for future research.

\section{DEFINITIONS AND PRELIMINARIES}

All graphs considered here will be finite, simple and connected. We refer the reader to Ref. [9] or [10] for all graph-theoretic terms used but not defined here.

A hexagonal system is a connected collection of congruent regular hexagons arranged in a plane in such a way that two hexagons are either completely disjoint or have one common edge. To each such system we assign a benzenoid graph, taking the vertices of hexagons as the vertices of the graph, and the sides of hexagons as the edges of the graph. The resulting graph is simple, planar, bipartite and all its bounded faces are hexagons. The name comes from the fact that such graphs are natural models for benzenoid hydrocarbons, a large and important class of organic compounds in which carbon atoms are arranged in 
cycles of length six. Benzenoids belong to a larger class called the polycyclic aromatic hydrocarbons (PAHs) which may contain cycles of different lengths, and some of the concepts defined here apply to all PAHs and to their mathematical models. However, we will restrict our attention here to alternant PAHs, i.e., PAHs with only rings of length six, and from now on the two terms (benzenoids and PAHs) will be used interchangeably.

A matching in a graph $G$ is a collection $M$ of edges of $G$ such that no two edges from $M$ share a vertex. A matching $M$ is perfect if every vertex of $G$ is incident with exactly one edge from $M$.

Perfect matchings in benzenoid and in related graphs are often called Kekule structures in the chemical literature. The benzenoids with perfect matchings are called Kekuléan. The literature on the enumeration and on other aspects of perfect matchings in benzenoids is vast; the reader might wish to see, e.g., Ref. [3] and the references therein.

The superposition of the two Kekule structures in benzene is called the Clar's aromatic sextet. In larger benzenoids, more than two Kekulé structures are needed to describe the resonance patterns. A Clar structure is a Kekule structure with the largest possible number of disjoint aromatic sextets. The largest possible number of disjoint aromatic sextets in a structure is its Clar number. According to the Clar's rule, ${ }^{[1,2,11]}$ Clar structures are the most important structures for explaining the properties of benzenoid compounds. Aromatic sextets are usually depicted as circles in the corresponding hexagons.

For a given polycyclic aromatic hydrocarbon its standard structure, as defined in Ref. [17], is defined as a structure that has the smallest possible number of unpaired electrons and does not have aromatic sextets.

In a Kekuléan benzenoid, any Kekulé structure (and the corresponding perfect matching) can serve as a standard one. In non-Kekuléan benzenoids, standard structures are necessarily represented by non-perfect matchings. The number of unpaired electrons corresponds to the defect of that matching, i.e., to the number of vertices not incident with edges of the matching. Examples of standard structures in a Kekuléan and in a non-Kekuléan benzenoid are shown in Figures 1. and 2., respectively.

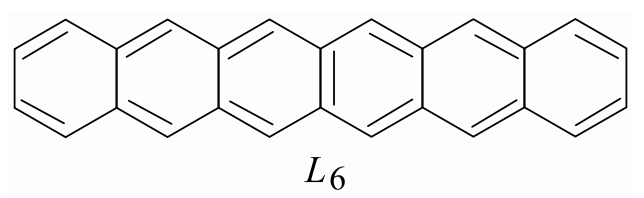

Figure 1. A standard structure in a linear polyacene.

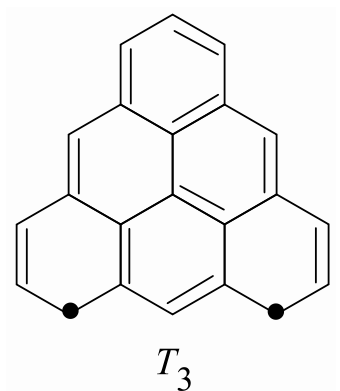

Figure 2. A standard structure in a non-Kekuléan benzenoid.<smiles>c1ccccc1</smiles>

$-90 \mathrm{~kJ} / \mathrm{mol}$

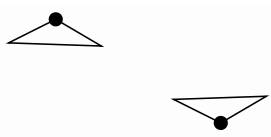

$+272 \mathrm{~kJ} / \mathrm{mol}$
Figure 3. Energy penalties.

The energy of a standard structure of molecule $\alpha$ is denoted by $E_{\alpha}$ and expressed in $\mathrm{kJ} / \mathrm{mol}$. As the aromatic stabilization energy of benzene has been reported as 90 $\mathrm{kJ} / \mathrm{mol},{ }^{[14]}$ to a structure with $k$ extra aromatic sextet the energy of $E_{\alpha}-k \cdot 90 \mathrm{~kJ} / \mathrm{mol}$ is assigned. Similarly, since the $\mathrm{C}-\mathrm{C} \pi$-bonding energy is estimated at about $272 \mathrm{~kJ} / \mathrm{mol},{ }^{[8]}$ any two extra unpaired electrons raise $E_{\alpha}$ by $272 \mathrm{~kJ} / \mathrm{mol}$. Hence, according to Ref. [17], a structure with $k$ extra aromatic sextets and $2 /$ unpaired electrons is assigned energy of $E_{\alpha}-k \cdot 90+I \cdot 272 \mathrm{~kJ} / \mathrm{mol}$. The energy penalties are shown schematically in Figure 3.

The structures with the smallest energy are called the most important structures. The structures with the second smallest energy are called the second most important structures, and so on. The number of structures with the same energy is called the degeneracy of that structure.

The degeneracy of the $i$-th most important structure of a graph $G$ we denote by $\Delta_{i}(G)$.

The degeneracies of the most important and the second most important structure of anthracene are shown in Figure 4.

Next we introduce some well-known and wellresearched combinatorial families which will be brought to bijective correspondences with our most important structures.

A Dyck path on $2 n$ steps is a lattice path in the coordinate plane $(x, y)$ from $(0,0)$ to $(2 n, 0)$ with steps $(1,1)$ $(U p)$ and $(1,-1)(D o w n)$, never falling below the $x$-axis. The number of steps is called the length of the path. The set of all Dyck paths of length $2 n$ we denote by $\mathcal{D}(n)$. A Dyck path of length 14 is shown in Figure 5.

The lattice paths from $(0,0)$ to $(2 n, 0)$ constructed by using the same steps but omitting the restriction of never 
<smiles>c1ccc2cc3ccccc3cc2c1</smiles><smiles>c1ccc2cc3ccccc3cc2c1</smiles><smiles>c1ccc2c(c1)CC1Cc3ccccc3C21</smiles><smiles>c1ccc2cc3ccccc3cc2c1</smiles>

$$
\Delta_{2}\left(L_{3}\right)=1
$$

Figure 4. Examples of degeneracies.

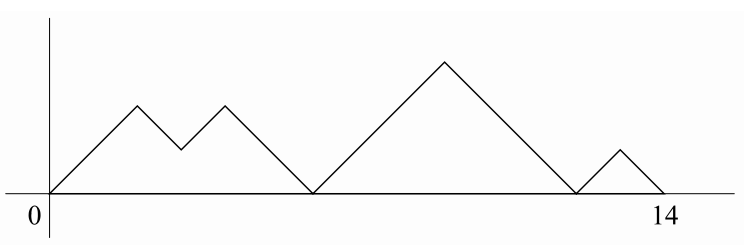

Figure 5. A Dyck path from $\mathcal{D}_{4}(7)$.

falling below the $x$ axis are called grand Dyck paths. They stand in a bijective correspondence with Kekule structures in certain classes of benzenoid graphs; see Refs. [6] and [7] for explicit constructions and some consequences. For another appearance of Dyck paths in a chemical setting see Ref. [5].

A peak of a Dyck path is a place where an Up step is immediately followed by a Down step. The set of all Dyck paths of length $2 n$ with exactly $k$ peaks we denote by $\mathcal{D}_{k}(n)$. Obviously, $\mathcal{D}_{k}(n) \cap \mathcal{D}_{l}(n)=\varnothing$ for $k \neq l$ and $\mathcal{D}(n)=\bigcup_{1 \leq k \leq n} \mathcal{D}_{k}(n)$.

In a similar way, motivated by the obvious resemblance of Dyck paths to mountain landscapes, we define valleys as the places where a Down step is followed by an Up step, and ascents and descents as consecutive sequences of $U p$ and Down steps, respectively.

There are several ways to prove that the Dyck paths on $2 n$ steps are enumerated by the Catalan numbers $C_{n}=\frac{1}{n+1}\left(\begin{array}{c}2 n \\ n\end{array}\right)$. This sequence enumerates many other combinatorial families. Some seventy of them are listed in Ex. 6.19 of Stanley's book, ${ }^{[15]}$ and there are many more 214 families are listed in. ${ }^{[16]}$ The best known of these families are triangulations of a convex $(n+2)$-gon, binary trees with $n$ vertices, plane trees with $n+1$ vertices, $n$ nonintersecting chords connecting $2 n$ points on the circumference of a circle and the legal parenthesizations (bracketings) of a string of $n+1$ letters subject to a nonassociative binary operation. (A parenthesization is legal if it has equally many left and right parentheses, if, counting from left to right, the number of the right parentheses never exceeds the number of the left ones and if there are no empty pairs of parentheses.) The last interpretation will be particularly suitable as an intermediate step between our structures and Dyck paths.
There are many good references on Catalan numbers. A good starting point is the excellent recent monograph of Stanley. ${ }^{[16]}$

The Narayana numbers $N(n, k)$ are defined for integers $n, k \geq 1$ by

$$
N(n, k)=\frac{1}{n}\left(\begin{array}{l}
n \\
k
\end{array}\right)\left(\begin{array}{c}
n \\
k-1
\end{array}\right)=\frac{1}{k}\left(\begin{array}{c}
n \\
k-1
\end{array}\right)\left(\begin{array}{c}
n-1 \\
k-1
\end{array}\right),
$$

with the initial value $N(0,0):=1$ and the boundary values $N(n, 0)=0, N(n, 1)=1$ for $n \geq 1$. It is easy to see, by a direct computation, that the Narayana numbers decompose the Catalan numbers, i.e. that $\sum_{k \geq 0} N(n, k)=C_{n}$, for all $n \geq 0$, and that this decomposition is the one defined by the number of peaks in Dyck paths. In other words, the Narayana numbers $N(n, k)$ enumerate Dyck paths on $2 n$ steps with exactly $k$ peaks.

\section{Proposition 2.1.}

$$
\left|D_{k}(n)\right|=N(n, k)
$$

For a combinatorial proof of this result, we refer the reader to the article Ref. [4]. The Narayana numbers have many other interesting properties. The most interesting for us will be their symmetry,

$$
N(n, k)=N(n, n+1-k),
$$

in particular for $k=2$, i.e., the equality $N(n, n-2)=N(n, 3)$.

\section{MAIN RESULTS}

In this section we provide explicit formulas for degeneracies of the most important structures in four classes of benzenoids. The first two considered classes are special types of chains, the third one belongs to catacondensed benzenoids (with no vertices shared by three hexagons), while the last one represents pericondensed benzenoids.

\section{Linear Polyacene}

We first consider linear polyacenes $L_{n}$ consisting of $n$ hexagons. An example is shown in Figure 6. The two hexagons 
<smiles>c1ccc2cc3cc4cc5ccccc5cc4cc3cc2c1</smiles>

Figure 6. One of the $n$ most important structures in a linear polyacene.

adjacent to only one other hexagon are called terminal hexagons; all others, if any, are internal. (The term "internal hexagon" has a different meaning in the context of pericondensed benzenoids, where it denotes a hexagon surrounded by six other hexagons.) It has been well known for a long time that linear polyacenes are Kekuléan and that their Clar number is equal to one. Since all their most important structures have exactly one aromatic sextet and this sextet can be located in any of their $n$ hexagons, it follows that the degeneracy of the most important structures in $L_{n}$ is equal to the number of hexagons $n$.

\section{Proposition 3.1.}

$$
\Delta_{1}\left(L_{n}\right)=n
$$

The situation is more interesting for the second most important structures. All such structures have two unpaired electrons and two aromatic sextets. We first prove an auxiliary result on their arrangement in $L_{n}$.

\section{Lemma 3.2.}

The unpaired electrons in a second most important structure of $L_{n}$ must belong to internal hexagons. Both must be at vertices of degree 2, and one of them must be on the upper part of the border, while the other must be on the lower part.

Proof. Let $u$ and $v$ be the vertices of $L_{n}$ corresponding to the unpaired electrons. Then the set $\{u, v\}$ must be independent (i.e., $u$ and $v$ cannot be adjacent) and nice (i.e., the graph $L_{n} \backslash\{u, v\}$ must contain a perfect matching). Moreover, there must be a perfect matching in $L_{n} \backslash\{u, v\}$ with exactly two aromatic sextets, so that the energy fits the requirements of the second most important structure. An example is shown in Figure 7.

Let us embed $L_{n}$ into the plane so that the edges shared by adjacent hexagons are vertical. Then all vertices of degree 3 are on vertical edges, half of them at their upper ends, half on their lower ends. Moreover, all vertices of degree 3 on the upper side of vertical edges belong to the same class of bipartition, and all vertices of degree 3 on the lower side belong to the opposite class.

Let us suppose that $u$ and $v$ are both of degree 3 . Then one of them, say $u$, lies on the upper border of $L_{n}$, and $v$ lies on the lower border. As they cannot be adjacent,<smiles></smiles>

Figure 7. A second most important structure in a linear polyacene.<smiles>C1=Cc2cc3ccccc3cc2C=c2cc3cc4cc5ccccc5cc4cc3cc2=C1</smiles>

Figure 8. Unpaired electrons at vertices of degree 3.

let us suppose that $u$ lies to the left of $v$, as shown in Figure 8 . The total number of edges on the boundary of $L_{n}$ is $4 n+2$ and they form the cycle $C_{4 n+2}$. It follows by a simple parity argument that the distance between $u$ and $v$ along this cycle is odd, due to the odd number of edges in terminal hexagons. Hence the removal of $u$ and $v$ splits the $C_{4 n+2}$ into two even paths, both of them having a perfect matching. The union of those two perfect matchings is a perfect matching in $L_{n} \backslash\{u, v\}$. It is easy to see that this is the only perfect matching in $L_{n} \backslash\{u, v\}$. Indeed, the same parity argument implies that no vertical edge of $L_{n} \backslash\{u, v\}$ can be in a perfect matching of this graph. Hence, there cannot be an aromatic sextet in $L_{n} \backslash\{u, v\}$, contrary to our assumption.

Next we consider the case that $u$ is of degree 3 and $v$ of degree 2. Then, with one exception, both of them must be either on the upper or on the lower part of the border. (Otherwise they would belong to the same class of bipartition.) Let they be both on the upper part; see Figure 9. It follows by the same parity argument that no vertical edge lying between $u$ and $v$ can participate in any perfect matching of $L_{n} \backslash\{u, v\}$. The same is valid for any vertical edge lying between $u$ and the terminal hexagon closer to it than to $v$. The vertical edges between $v$ and the terminal hexagon closer to it than to $u$ can participate in perfect matchings. Any such matching, however, can have only one aromatic sextet and cannot have the energy of the second most important structure. The exception mentioned above is the case when the vertex of degree 2 lies on the vertical<smiles></smiles>

Figure 9. Unpaired electrons at vertices at the same part of the border. 


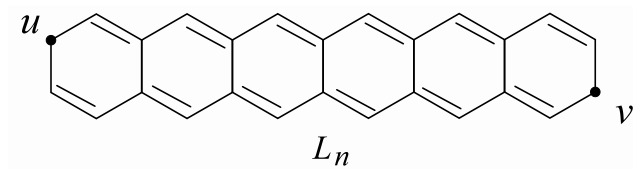

Figure 10. Unpaired electrons at vertices of degree 2 on vertical edges.

edge in one of the two terminal hexagons. Then it must be on the opposite part of the border. In our case, with $u$ on the upper part, $v$ must be on the lower part. Again, all edges are forced and hence no perfect matching of $L_{n} \backslash\{u, v\}$ can contain an aromatic sextet, let alone two.

It remains to consider the case when both unpaired vertices are of degree 2 . If both of them lie on vertical edges, they cannot be both on the same edge, so one of them must be on the leftmost and the other one on the rightmost vertical edge. If both are on the upper border, then they must belong to the same class of bipartition, and the rest of $L_{n}$ cannot have a perfect matching. Let the unpaired vertex on the leftmost vertical edge be on the upper border of $L_{n}$ as in Figure 10. Then the other unpaired vertex must be the lower one on the rightmost vertical edge. Then all edges of the unique perfect matching in the rest of the graph are forced and the unique perfect matching cannot have an aromatic sextet. This is a contradiction with our assumption that the considered structure is a second most important one.

If only one of them, say $u$, lies on a vertical edge, then the other one must lie on the same part of the border. Then all edges between $u$ and $v$ are forced, and the same argument as above implies that only the part of $L_{n}$ to the right of $v$ can contain aromatic sextets. Since it is straight, it contains exactly one aromatic sextet. It follows that no perfect matchings in $L_{n} \backslash\{u, v\}$ contains two aromatic sextets. Hence none of $u, v$ lies on vertical edges.

Let $u$ lie on the top of hexagon $k$ and $v$ on the bottom of hexagon I with $k \geq I$. Then all edges of any perfect matching in $L_{n} \backslash\{u, v\}$ lying in hexagons $k, \ldots, l$ are forced, leaving the two parts outside hexagons $k$ and $I$ to each contain one aromatic hexagon. Hence, none of $u$ and $v$ can lie on terminal hexagons.

The remaining cases follow by symmetry.

Lemma 3.2 will be useful also for other considered catacondensed cases.

Theorem 3.3.

$$
\Delta_{2}\left(L_{n}\right)=\frac{1}{6}\left(\begin{array}{c}
(n-1)^{2} \\
2
\end{array}\right)
$$

Proof. Let us take a second most important structure in $L_{n}$. As noted above, it must have exactly two aromatic sextets and exactly one pair of unpaired electrons. Moreover, from Lemma 3.2 we know that all unpaired electrons must lie between two aromatic hexagons. For each such structure we construct a legal bracketing with two pairs of parentheses in a string of $n-2$ letters in the following way. We scan the structure from left to right. To each nonaromatic hexagon we assign a letter. To the first aromatic hexagon we assign a left parenthesis. To a hexagon with an unpaired electron on its top, we assign a left parenthesis and a letter; to a hexagon with an unpaired electron at its bottom, we assign a letter and a right parenthesis. If there is a hexagon containing two unpaired electrons, we assign to it a letter enclosed in a pair of parentheses. Finally, to the second aromatic sextet we assign a right parenthesis. It follows from Lemma 3.2 than any such bracketing must be legal, and the correspondence is clearly bijective. It is illustrated in the left-hand side of Figure 11. for $L_{4}$. The right-hand side of the same figure illustrates the canonical bijection between bracketings and Dyck paths: Left parentheses correspond to the Up steps, the right ones to the Down steps and letters correspond to peaks. It is now clear that the number of the second most important structures, i.e., their degeneracy, is equal to the Narayana number $N(n, n-2)=N(n, 3)$ and our claim

$$
\Delta_{2}\left(L_{n}\right)=\frac{1}{6}\left(\begin{array}{c}
(n-1)^{2} \\
2
\end{array}\right)
$$

readily follows by rearranging the obtained expression.

Next we obtain explicit formulas for the degeneracy of the third most important structure in linear polyacenes. The smallest polyacene allowing such structures is the linear pentacene.

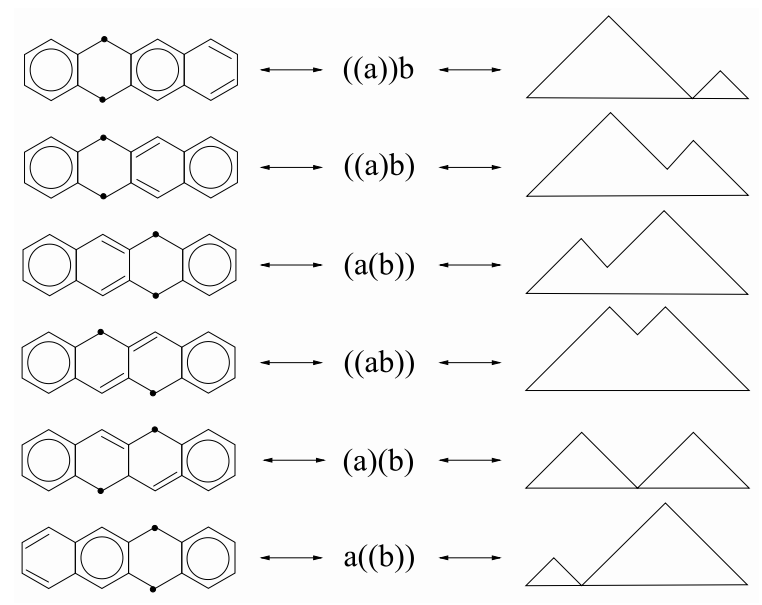

Figure 11. Bijection between second most important structures in a linear polyacene and Dyck paths. 
Theorem 3.4.

$$
\Delta_{3}\left(L_{n}\right)=\frac{2 n^{2}-8 n+11}{21}\left(\begin{array}{l}
n \\
5
\end{array}\right) .
$$

Proof. An example of a third most important structure in $L_{7}$ is shown in Figure 12. Any such structure must have three aromatic sextets and two pairs of unpaired electrons, two electrons on the upper and two on the lower part of the border. Counting such structures reduces to the problem of counting the number of ways of choosing three aromatic sextets and of positioning unpaired electrons between them. For a given $n$, the leftmost aromatic sextet in $L_{n}$ can be chosen in $n-4$ ways - it can be any of hexagons labeled by $1,2, \ldots, n-4$. Denote its location by $k$. Now the middle aromatic sextet can be placed in any hexagon from $k+2$ to $n-2$. Let it be hexagon $m$. Finally, the rightmost aromatic sextet can be placed in any hexagon between $m+2$ and $n$. The total number of ways of choosing three aromatic sextets in $L_{n}$ is given by

$$
\sum_{k=1}^{n-4} \sum_{m=k+2}^{n-2}(n-m-1)=\sum_{k=1}^{n-4}\left(\begin{array}{c}
n-k-2 \\
2
\end{array}\right)=\left(\begin{array}{c}
n-2 \\
3
\end{array}\right) .
$$

For two aromatic hexagons at distance $\ell \geq 2$ there are $(\ell-1)^{2}$ ways to insert two unpaired electrons, since they can be chosen independently of each other, one in each of $\ell-1$ available positions on the upper and on the lower border, respectively. The claim of the Theorem now follows by summing over all legal positions of three aromatic sextets and simplifying the resulting expressions:

$$
\sum_{k=1}^{n-4} \sum_{m=k+2}^{n-2}(m-k-1)^{2} \sum_{\ell=m+2}^{n}(\ell-m-1)^{2}=\frac{2 n^{2}-8 n+11}{21}\left(\begin{array}{l}
n \\
5
\end{array}\right) .
$$

Our results in this subsection provide explicit expressions for the values reported in Table 3 of Ref. [17]. They also provide correct values for the degeneracies of the third most important structures in $L_{18}, L_{19}$ and $L_{20}$ as 210120,321708 and 480624, respectively. Those values are incorrectly reported in the rightmost column of Table 3 of Ref. [17]. The sequence $\Delta_{2}\left(L_{n}\right)$ appears as sequence $A 002415$ in the On-Line Encyclopedia of Integer Sequences, ${ }^{[12]}$ while the sequence $\Delta_{3}\left(L_{n}\right)$, starting with $1,10,53,200,606, \ldots$, seems to be new. It would be interesting to investigate whether our results could be also relevant in the context of

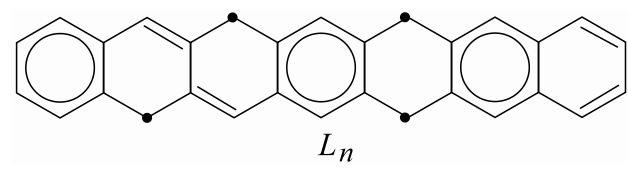

Figure 12. A third most important structure in a linear polyacene. lithium adsorption on polyacenes and on more general zigzag-edge graphene strips. ${ }^{[13]}$

\section{Straight Chains With one Kink}

In this subsection we consider benzenoid chains $L_{k, l}$ of length $k+I+1$ in which two straight polyacene segments of length $k$ and $l$, respectively, are joined by one kinky hexagon. (A hexagon is kinky if the two edges it shares with other hexagons are neither adjacent nor parallel.) An example is shown in Figure 13.

We take $k \geq l$. As the cases when both $k$ and $l$ are smaller than 3 were considered in Ref. [17], we assume $k \geq 3$. A segment of $L_{k, l}$ is long if its length is at least three.

The most important structures are Kekuléan with two aromatic sextets, one in each segment. There are $m$ ways to place an aromatic sextet in a segment of length $m$. Since the sextets can be placed independently of each other, we have altogether $k \cdot l$ most important structures.

\section{Proposition 3.5.}

Let $L_{k, l}$ be a one-kink benzenoid chain with both segments long. Then

$$
\Delta_{1}\left(L_{k, l}\right)=k \cdot l
$$

The second most important structure is unique. It is Kekuléan, with just one aromatic sextet in the kink hexagon. Hence, $\Delta_{2}\left(L_{k, l}\right)=1$

Since any Kekuléan structure in $L_{k, l}$ must have at least one aromatic sextet, it follows that any third most important structure must have two unpaired electrons. Their placement is still governed by Lemma 3.2. If the unpaired electrons are placed on different segments, there can be at most two aromatic sextets in the rest of the graph; if they are placed on the same segment, three aromatic sextets can be placed in the rest of the graph, resulting in a structure with lower energy. Since there is no way to place more than three aromatic sextets in $L_{k, l}$ after removal of any two vertices, any third most important structure must have exactly three aromatic sextets. Hence the unpaired electrons must be on the same segment, on the opposite parts of its border, and there must be three

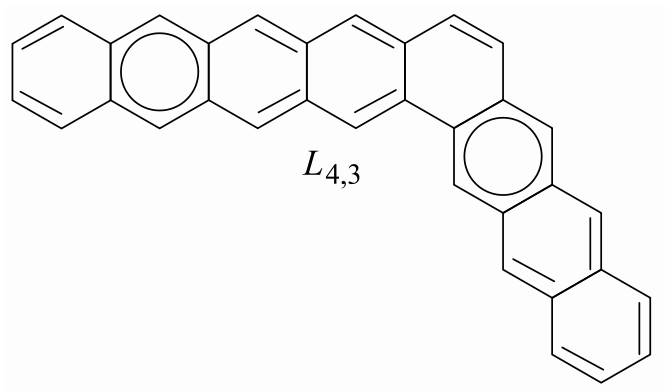

Figure 13. A one-kink chain with long segments. 
aromatic sextets, two on the segment with unpaired electrons, and the third one on the other segment. The following result is then a simple consequence of the results of the previous subsection.

\section{Proposition 3.6.}

Let $L_{k, l}$ be a one-kink benzenoid chain with both segments long. Then

$$
\Delta_{3}\left(L_{k, l}\right)=\Delta_{1}\left(L_{k}\right) \Delta_{2}\left(L_{l}\right)+\Delta_{2}\left(L_{k}\right) \Delta_{1}\left(L_{l}\right)=k \Delta_{2}\left(L_{l}\right)+I \Delta_{2}\left(L_{k}\right)
$$

By defining $\Delta_{2}\left(L_{1}\right)=0$ for $I<3$ the above result becomes valid also for one-kink chains in which only one segment is long.

\section{Branched Benzenoid with Straight Branches}

The simplest branched benzenoid is the triphenylene. We consider here a bit more general case with one branching hexagon and three straight segment of length $k, l$, and $m$. For the sake of simplicity, we assume that all segments are long. Such a structure is denoted by $S(k, I, m)$; an example is shown in Figure 14.

Since the Clar number of $S(k, I, m)$ is equal to 3 , the most important structures will be Kekuléan, with one aromatic sextet in each segment. The sextets can be chosen independently, and hence the number of such structures is equal to the product of the segment lengths.

\section{Proposition 3.7.}

Let $S(k, I, m)$ be a benzenoid with one branching hexagon and three long polyacene branches of length $k, l$, and $m$. Then $\Delta_{1}(S(k, I, m))=k l m$.

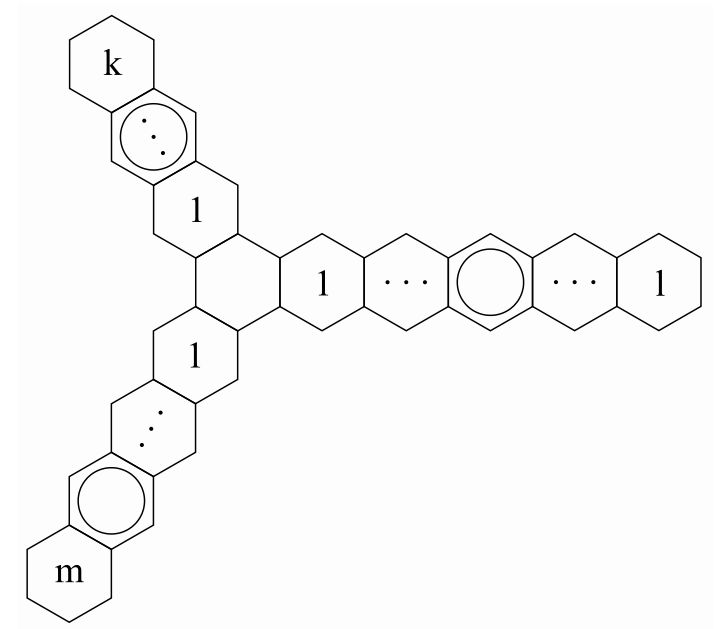

Figure 14. A branched catacondensed benzenoid with three long polyacene segments.
It follows by parity that a Kekuléan structure in $S(k, l, m)$ cannot have exactly two aromatic sextets, and that there is only one Kekuléan structure with exactly one aromatic sextet - the one with the aromatic sextet in the branching hexagon. As in the case of one-kink chains, this structure is the second most important. Hence $\Delta_{2}(S(k, l, m))=1$.

As before, any third most important structure must have two unpaired electrons, and by the same arguments we can conclude that the energetically most favorable placement must place both of them on the same branch. As all branches are long, it follows that the number of aromatic sextets must be four, two on the branch with unpaired electrons and one on each of the remaining branches. Now the degeneracy of the third most important structure can be compactly expressed in the following way.

\section{Proposition 3.8.}

Let $S(k, I, m)$ be a benzenoid with one branching hexagon and three long polyacene branches of length $k, l$, and $m$. Then

$$
\Delta_{3}(S(k, I, m))=k l m \sum_{p \in\{k, I, m\}} \frac{\Delta_{2}\left(L_{p}\right)}{\Delta_{1}\left(L_{p}\right)} .
$$

For short branches we have more complicated situations and we omit the detailed case-by-case analysis.

\section{Strips of a Fixed Width}

The last class we consider here are benzenoid strips (or benzenoid parallelograms) of varying length and fixed width. An example of length 6 and width 3 is shown in Figure 15. In this subsection we do not distinguish between various Kekuléan structures and consider only the most important non-Kekuléan structures. Their degeneracy will be denoted by $\Omega_{2}\left(B_{n, m}\right)$. An example is shown in Figure 16 . Our first results are explicit formulas for cases $m=2,3$.

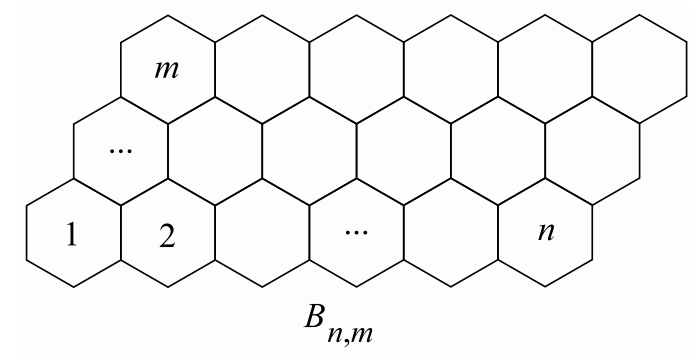

Figure 15. Benzenoid strip of length 6 and width 3 . 


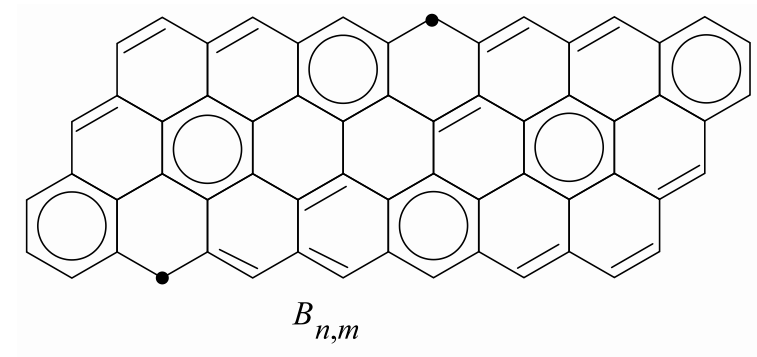

Figure 16. A most important non-Kekuléan structure in $B_{6,3}$.

Proposition 3.9.

$$
\begin{aligned}
& \Omega_{2}\left(B_{n, 2}\right)=\frac{5 n-1}{6}\left(\begin{array}{l}
n \\
5
\end{array}\right) ; \\
& \Omega_{2}\left(B_{n, 3}\right)=\frac{7 n^{2}+n-146}{28}\left(\begin{array}{l}
n \\
6
\end{array}\right) .
\end{aligned}
$$

Proof. Let us look at the case $m=2$. We must count the ways of choosing one aromatic sextet in the lower row, then one in the upper row, then again one in the lower row and finally one in the upper row. If the hexagons are numbered left to right by $1,2, \ldots, n$, their indices must differ by at least one between a lower one and the upper one, and at least by two between an upper one and the lower one following it. This is possible for all $n \geq 5$. If the chosen hexagons in the lower row are labeled by $k$ and $m$, and in the upper row by $I$ and $p$, we must have $k \leq I-1$, $I \leq m-2$ and $m \leq p-1$. There are $m-k-1$ ways to insert one unpaired electron on the lower border and $p-I-1$ way to do the same on the upper border. The claim now follows by summing over all possible arrangements of $k, l, m, p$ satisfying the above conditions:

$$
\sum_{k=1}^{n-4} \sum_{l=k+1}^{n-3} \sum_{m=l+2}^{n-1} \sum_{p=m+1}^{n}(m-k-1)(p-l-1)=\frac{5 n-1}{6}\left(\begin{array}{l}
n \\
5
\end{array}\right)
$$

The case $m=3$ follows along the same lines for $n \geq 6$. The number of nested sums increases to six, but only two summands are non-constant.

$$
\begin{aligned}
& \sum_{k=1}^{n-5} \sum_{l=k+1}^{n-4} \sum_{m=l+1}^{n-3} \sum_{p=l+2}^{n-2} \sum_{q=p+1}^{n-1} \sum_{r=q+1}^{n}(p-k-1)(r-m-1)= \\
& \frac{7 n^{2}+n-146}{28}\left(\begin{array}{l}
n \\
6
\end{array}\right)
\end{aligned}
$$

In general, for a fixed $m \geq 2, \Omega_{2}\left(B_{n, m}\right)$ is a polynomial of degree $2 m+2$ in $n$ obtained by evaluating the $2 m$-fold nested sum

$$
\begin{gathered}
\sum_{i_{1}=1}^{n-m-2} \sum_{i_{2}=i_{1}+1}^{n-m-1} \cdots \sum_{i_{m}=i_{m-1}+1}^{n-3} \sum_{i_{m+1}=i_{2}+2}^{n-m-1} \sum_{i_{m+2}=i_{m+1}+1}^{n-m} \cdots \\
\sum_{i_{2 m}=i_{2 m-1}+1}^{n}\left(i_{m+1}-i_{1}-1\right)\left(i_{2 m}-i_{m}-1\right) .
\end{gathered}
$$

For $m=1$, the above expression reduces to our previously derived formula,

$$
\Omega_{2}\left(B_{n, 1}\right)=\frac{2(n-1)}{n-3}\left(\begin{array}{l}
n \\
4
\end{array}\right)=\Delta_{2}\left(L_{n}\right) .
$$

\section{OPEN PROBLEMS AND CONCLUDING REMARKS}

In this contribution we have counted the most important structures in several classes of benzenoid graphs and thus provided explicit expressions for their degeneracies. Our methods could be used to solve the same problem in various other classes of benzenoids and of more general graphs. In this sections we indicate some possible candidates.

One could start with benzenoid chains with kinks. We have presented some initial results for chains with two long straight segments separated by one kink, but for other cases the problem is still open. Branched catacondensed benzenoids would be the next logical choice, followed by pericondensed benzenoids of high symmetry. Some of such structures have been investigated in Ref. [17], but the results are far from satisfactory. For example, the degeneracy of the second most important structure in the rhombic graphene nanoflake shown in Figure 17. has been reported as 18. Indeed, for the arrangement of aromatic sextets shown in Figure 17., nine structures with the same energy can be obtained by positioning one unpaired electron at any of the places indicated by arrows in the

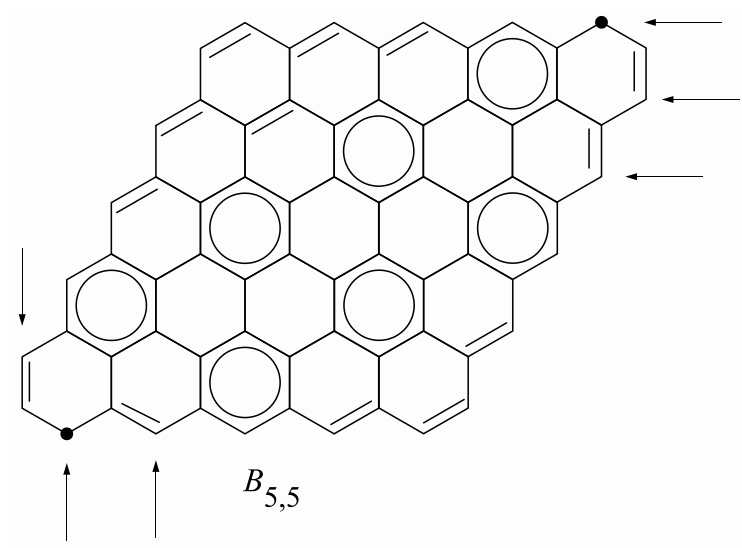

Figure 17. 18 second most important structures in a rhombic nanoflake found in Ref. [17]. 


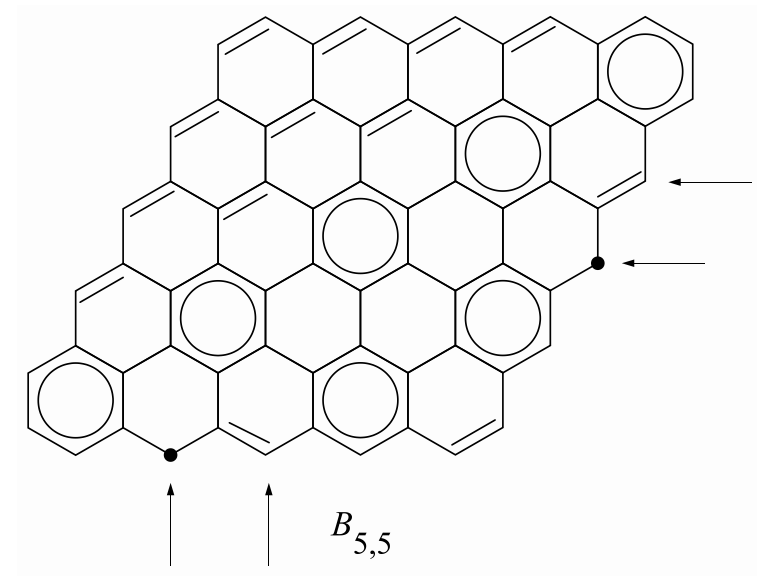

Figure 18. More second most important structures in the same rhombic nanoflake.

upper right corner and the other one on any of places similarly indicated in the lower left corner, and another nine structures could be obtained by mirroring the aromatic sextet arrangement across the long diagonal. There are, however, more structures of the same energy. Eight more of them can be obtained in the way indicated in Figure 18.

We leave to the interested reader to derive the exact expression as a function of the length $n$ of the rhombus side.

It would be also interesting to consider such structures on nanoflakes with defects, in particular on nanocones and fullerene patches. Finally, one could also formulate and investigate analogous problems for lattice animals on other lattices.

Acknowledgment. Partial support of the Croatian Science Foundation via research project LightMol (Grant no. HRZZ-IP2016-06-1142) and of the Slovenian research agency ARRS, program no. P1-0383, is gratefully acknowledged. I thank all referees for careful reading and insightful suggestions.

\section{REFERENCES}

[1] E. Clar, The Aromatic Sextet, J. Wiley and Sons, 1972.

[2] E. Clar, Polycyclic Hydrocarbons, Academic Press, 1964. https://doi.org/10.1007/978-3-662-01665-7

[3] S. J. Cyvin, I. Gutman, Kekulé Structures in Benzenoid Hydrocarbons, Lecture Notes in Chemistry 46, Springer, Heidelberg, 1988.

https://doi.org/10.1007/978-3-662-00892-8

[4] E. Deutsch, Discrete Math. 1999, 204, 167-202. https://doi.org/10.1016/S0012-365X(98)00371-9

[5] T. Došlić, Croat. Chem. Acta 2002, 75, 881-889.

[6] T. Došlić, Croat. Chem. Acta 2005, 78, 251-259.

[7] T. Došlić, Math. Magazine 2007, 80, 219-226. https://doi.org/10.1080/0025570X.2007.11953485

[8] J. E. Douglas, B. S. Rabinovitch, F. S. Looney, J. Chem. Phys. 1955, 23, 315-323.

https://doi.org/10.1063/1.1741959

[9] F. Harary, Graph Theory, Addison-Wesley, Reading MA, 1969. https://doi.org/10.21236/AD0705364

[10] Lovász, L. and M. D. Plummer, Matching Theory, Ann. Discr. Math. 29, North-Holland, Amsterdam, The Netherlands, 1986.

[11] A. Misra, D. J. Klein, T. Morikawa, J. Phys. Chem. A 2009, 113, 1151-1158. https://doi.org/10.1021/jp8038797

[12] OEIS Foundation Inc., The On-Line Encyclopedia of Integer Sequences, https://oeis.org/.

[13] Y. P. Ortiz, D. J. Klein, T. H. Seligman, arXiv:1603.09308v1

[14] S. W. Slayden, J. F. Liebman, Chem. Rev. 2001, 101, 1541-1566. https://doi.org/10.1021/cr990324+

[15] R. P. Stanley, Enumerative combinatorics, Vol. 2, Cambridge University Press, Cambridge, 1999. https://doi.org/10.1017/CBO9780511609589

[16] R. P. Stanley, Catalan Numbers, Cambridge University Press, Cambridge, 2015. https://doi.org/10.1017/CBO9781139871495

[17] C.-N. Yeh, J.-D. Chai, Sci. Rep. 2016, 6, 30562. https://doi.org/10.1038/srep30562 